\title{
Clinical Management of Neuropsychiatric Symptoms of Huntington Disease: Expert-Based Consensus Guidelines on Agitation, Anxiety, Apathy, Psychosis and Sleep Disorders
}

Karen E. Anderson ${ }^{\mathrm{a}, *}$, Erik van Duijn ${ }^{\mathrm{b}}$, David Craufurd ${ }^{\mathrm{c}, \mathrm{d}}$, Carolyn Drazinic $^{\mathrm{e}}$, Mary Edmondson ${ }^{\mathrm{f}}$, Nathan Goodman ${ }^{\mathrm{g}}$, Daniel van Kammen ${ }^{\mathrm{h}}$, Clement Loy ${ }^{\mathrm{i}}$, Josef Priller ${ }^{\mathrm{j}}$ and LaVonne Veatch Goodman ${ }^{\mathrm{g}}$

${ }^{a}$ Department of Psychiatry and Department of Neurology, Georgetown University, Washington, DC, USA

${ }^{\mathrm{b}}$ Department of Psychiatry, Leiden University Medical Centre, Leiden; and Mental Health Care Centre Delfland, Delft, The Netherlands

${ }^{\mathrm{c}}$ Manchester Centre for Genomic Medicine, Division of Evolution and Genomic Sciences, School of Biological Sciences, Faculty of Biology, Medicine and Health, University of Manchester, Manchester Academic Health Science Centre, Manchester, UK

'St Mary's Hospital, Central Manchester University Hospitals NHS Foundation Trust, Manchester Academic Health Science Centre, Manchester, UK

${ }^{\mathrm{e}}$ Chief Medical Officer of State Mental Health Facilities, Office of Substance Abuse and Mental Health Florida Department of Children and Families, Tallahassee, FL, USA

${ }^{\mathrm{f}} \mathrm{HD}$ Reach, Raleigh, NC, USA

${ }^{\mathrm{g}}$ Huntington's Disease Drug Works, Seattle, WA, USA

${ }^{\mathrm{h}}$ Consultant for CNS drug development, Professor emeritus University of Pittsburgh, Pittsburgh, PA, USA

${ }^{\mathrm{i}}$ Westmead Huntington Disease Service, The University of Sydney, and the Garvan Institute of Medical Research, Sydney, Australia

${ }^{\mathrm{j} D e p a r t m e n t ~ o f ~ N e u r o p s y c h i a t r y, ~ C h a r i t e ́ ~-~ U n i v e r s i t a ̈ t s m e d i z i n, ~ B e r l i n, ~ G e r m a n y ~ a n d ~ U n i v e r s i t y ~ o f ~ E d i n b u r g h ~}$ and UK DRI, Edinburgh, UK

\footnotetext{
Abstract.

Background: In clinical practice, several strategies and pharmacological options are available to treat neuropsychiatric symptoms of Huntington disease (HD). However, there is currently insufficient data for evidence-based guidelines on the management of these common symptoms.
}

\footnotetext{
*Correspondence to: Karen E. Anderson, Georgetown University, Psychiatry Research Division, Suite 120,2115 Wisconsin Ave
} 
Objective: We aimed to develop expert-based recommendations regarding the management of agitation, anxiety, apathy, psychosis, and sleep disorders.

Methods: Guideline development was based on a modified Institute of Medicine guideline process that accounted for a lack of evidence base. An international committee of 11 multidisciplinary experts proposed a series of statements regarding the description and management of each symptom. Statement assessment and validation was performed using a web-based survey tool and 84 international HD experts (neurologists and psychiatrists) who assessed the statements and indicated their level of agreement.

Results: High-level agreement ( $\geq 85 \%$ experts strongly agreed or agreed) was reached for 107 of the 110 statements that have been incorporated into the expert-based clinical recommendations presented herein.

Conclusions: Clinical statements to guide the routine management of agitation, anxiety, apathy, psychosis, and sleep disorders in HD have been developed. Although not specifically tested in the HD population, clinical experience has shown that most of the neuropsychiatric symptoms discussed, when considered in isolation are treatable using pharmacologic and nonpharmacologic strategies developed for use in other populations. However, the management of neuropsychiatric symptoms in HD can be complex because neuropsychiatric symptoms often co-exist and treatment decisions should be adapted to cover all symptoms while limiting polypharmacy.

Keywords: Agitation, anxiety, apathy, clinical management, expert opinion, guidelines, Huntington disease, psychosis, sleep disorders

\section{INTRODUCTION}

Changes in behavior and mental state are a central feature of Huntington disease (HD) and often cause considerable distress and difficulty to patients, their relatives and other carers (caregivers) [1-3]. The neuropsychiatric and behavioral signs and symptoms of HD (henceforth referred to as neuropsychiatric symptoms for ease of reading) encompass the full spectrum of psychiatric illness [4, 5], and most people with HD will experience multiple neuropsychiatric symptoms or disorders during the course of their illness $[2,6]$. These can be related to the underlying progressive neurodegeneration of the brain but can also be a psychological reaction to the knowledge of being a gene expansion carrier and the impairments of the disease [7]. They may also arise as a side-effect of some HD medications. For example, some agents used to treat chorea may worsen apathy, depression, and other psychiatric symptoms $[8,9]$.

Over the past decade several comprehensive systematic reviews have been performed for the symptomatic treatment of HD and all have found an insufficient evidence base for the management of neuropsychiatric symptoms [10-12]. Although there is currently no cure for $\mathrm{HD}$, it is a misconception that the neuropsychiatric symptoms of HD are untreatable. Clinical experience has shown that treatments are available that can alleviate the neuropsychiatric symptoms of the disease. In the absence of a strong evidence based study, expert opinion obtained by methods to rigorously develop consensus is an acceptable option for developing clinical practice guidelines [14].

We have previously published opinion-based algorithms for the clinical management of chorea [15], irritability [16] and obsessive-compulsive or perseverative behaviors [17]. We aimed to expand this effort to encompass the management of agitation, anxiety, apathy, psychosis, and sleep disorders. While this work remains opinion based, we followed a more rigorous development process than employed for the earlier guides.

\section{MATERIALS AND METHODS}

Guideline development was based on a modified Institute of Medicine (IOM) guideline process [14] that accounted for a lack of a robust evidence base for these symptoms as they present in HD. The guideline development process began in November 2014 and was completed in May 2017. In line with the IOM process, an international core committee of 11 multidisciplinary experts was convened to lead guideline development. The core committee included 7 psychiatrists, 1 neurologist and 1 dually trained psychiatrist/neurologist drawn from behavioral working group committees of the European Huntington's Disease Network (EHDN) and the Huntington Study Group (HSG), an internal medicine physician, and a data analysis computer scientist. Two of the clinician experts are also family members of people with HD. 
In the first step of the process, core committee members independently developed a total of 493 statements (59-112 per symptom) for each of the five symptoms across the following IOM defined domains:

1. Definition of the symptom as it occurs in HD

2. Clinical description of the symptom and differential diagnosis

3. Prevalence

4. Preventive strategies

5. Treatment alternatives including behavioral and pharmaceutical options and addressing benefit, risks, and impact on coexisting symptoms for each treatment option

At this planning stage, committee members met face to face and by email, to combine redundant statements and discard inappropriate ones. A total of 110 statements were prepared for survey testing. For ease of reading, it was agreed to use the terminology of 'carer' in statements to refer the diverse group of people who support and care for people with HD, including (but not restricted to) partners, relatives, friends and paid carers (e.g., in nursing homes). It is acknowledged that not all people with HD will have a carer, for example if they are pre-symptomatic or are living with early stage HD.

Statement assessment and validation was performed using a web-based survey tool (SurveyMonkey). Five separate symptom surveys: agitation, anxiety, apathy, psychosis, and sleep disorders respectively, were uploaded for assessment and 120
HD experts (neurologists and psychiatrists) from the ongoing international Enroll-HD study platform [18] were invited to assess the statements and indicate their level of agreement.

Respondents could opt out of reviewing symptom domains if the symptom was outside of their expertise. The surveys employed a Likert scale with terms 'strongly agree', 'agree', 'disagree', 'strongly disagree', and 'insufficient experience'. Free text comments were also permitted. High-level consensus was declared when at least $85 \%$ of the respondents agreed (strongly agreed or agreed) with the statement. The number of respondents could differ per statement if respondents had opted out due to insufficient experience. Statements that did not reach a high-level consensus on the first round were combined, revised or deleted, and then sent back to the wider group for validation.

\section{RESULTS}

There was a 65-69\% response rate (depending on experience with each symptom) to the first surveys, with 84 unique respondents. Overall, 78 respondents from Australia, Europe and North America completed all five surveys. There was high level consensus for 98 of the 110 original statements $(89 \%)$, and these were retained for the final guidelines. The level of agreement for each of the original statements (by symptom domain) is provided in the Supplementary Material. Figure 1 shows the overall levels of agreement for the statements in each domain. Twelve

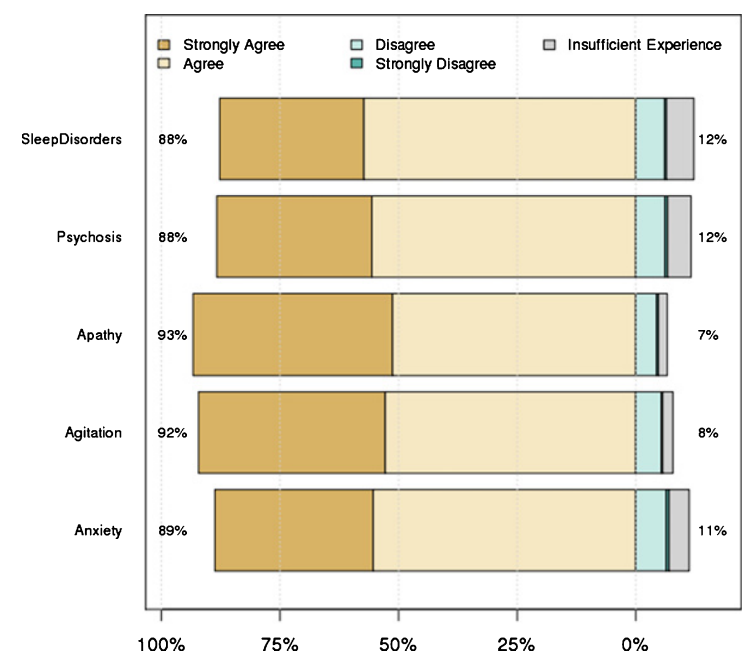

Fig. 1. Level of agreement with statements in each symptom domain. Numbers on left hand side represent the percent of people who strongly agreed or agreed, while the numbers on the right hand side represent the remainder. 
Table 1

\section{General Management for Symptoms of HD}

Assessment Recommendations
Obtain information about the symptom from the individual with HD when possible,
and collateral information from carers.
Identify coexisting psychiatric symptoms of HD that can contribute to the
presenting symptom.
Identify comorbid medical conditions that may contribute to the presenting
symptom of HD.
Review medications that may contribute to the presenting symptom.
Identify environmental factors that may contribute to the presenting symptom or its
severity.
Information Recommendations
Provide educational information about the nature and presentations of the symptom
in HD.

Provide information about methods that may be helpful for modifying symptom triggers.

\section{General Pharmacologic Recommendation}

Drug choice in HD should be influenced by coexisting symptoms and stage of disease.

Consult with a psychiatrist with knowledge of HD for those individuals who are resistant to standard pharmacologic treatment.

Regularly reassess continued need of drugs and potential for dose reduction because many of the adverse effects are difficult to distinguish from aspects of disease progression.

statements failed to reach strong consensus during the first round of survey review (Supplementary Material). The core committee reviewed all 12 statements and produced a follow-up survey containing nine revised statements; three statements were deleted. A total of 55 of the 84 original respondents completed the follow-up survey and all revised statements reached high-level consensus for inclusion in the final guidelines.

Statements pertaining to definition, clinical description and prevalence (IOM domains 1-3) are presented in narrative form, and statements regarding clinical practice recommendations for symptom prevention and management (IOM domain 4 and 5) have been combined and summarized by the expert committee and are presented in Tables 1-6. Where there were commonalities in the management of the five symptoms, the statements have been summarized as 'general principles' (Table 1). Representations of the clinical practice recommendations are presented in each section.

\section{Agitation in HD: Consensus definition and clinical description}

Agitation can occur at any time during the course of HD. Agitation in HD is generally defined as observable, situation-inappropriate behavior that is characterized by excessive motor or verbal activity and may manifest as a wide variety of behaviors, 
Table 2

\title{
Clinical Practice Guidelines for Agitation in HD
}

\author{
General Recommendations \\ Identify and treat comorbid medical conditions that can precipitate acute agitation \\ that include infectious, metabolic, toxic, drug-related, substance use, or other \\ medical causes of acute psychosis/delirium.
}

Promptly treat irritability or other coexisting psychiatric symptoms and sleep disturbances in HD as preventative strategy for agitation.

Modify environmental factors that can contribute to agitation including excessive noise or other overstimulation, pain and other unmet comfort needs, and misperceived threats.

\section{Behavioral Recommendations}

Provide educational information to carers about behavioral strategies that may lessen or prevent agitation behaviors.

When not a threat to self or others, the preferred initial response is to provide a safe, quiet space, time to calm down, and gentle verbal support.

\section{Pharmacologic Recommendations}

For acute agitation that is not responsive to behavioral strategies, the preferred pharmacologic options include use of either a benzodiazepine or an antipsychotic drug.

For chronic agitation characterized by recurrent and ongoing distress, or continuing threat of harm to self or others pharmacologic options include either an antipsychotic or a mood-stabilizing antiepileptic drug.

Consider a trial of pain medication when other therapies have failed for agitation in individuals who are unable to verbally communicate cause of distress.

including physical and verbal aggressive and nonaggressive behavior, general restlessness, pacing or screaming. Agitation can be a manifestation of HD, but other factors that can cause or exacerbate agitation in HD need to be considered. It can be caused by disease factors (e.g., progressive difficulty performing a motor or cognitive task, or impaired ability to communicate needs such as hunger or thirst, or discomfort and pain), but also co-existing psychiatric factors (e.g., obsessive and perseverative behaviors), co-morbid medical factors (e.g., metabolic, infectious, toxic, traumatic, prescription drug-related, substance use), and external environmental factors (e.g., change in routine, change in carer, excessive noise or being asked questions too quickly). Disinhibition can make the agitation worse.
Agitation may also be confused with akathisia (motor restlessness), a side effect of dopamine blocking medications.

The experts agreed that prompt treatment of coexisting psychiatric symptoms, co-morbid medical conditions and physical discomfort/pain may prevent episodes of agitation in HD. Depending on the situation, chronicity and severity of agitation, the experts recommended various medications that can be considered (Table 2). However, they noted that drugs commonly used for the treatment of agitation in HD are associated with adverse effects that may mimic symptoms of HD, such as apathy, slowing of cognitive processing, hypokinesia and tardive dyskinesia, swallowing impairment, and worsening of balance leading to falls. 
Table 3

\section{Clinical Practice Guidelines for Anxiety in HD}

\section{General Recommendations}

Treat coexisting psychiatric symptoms or comorbid medical conditions that can contribute to anxiety.

Modify environmental factors that can contribute to anxiety.

\section{Behavioral Recommendation}

Offer psychological behavioral therapy as first step in treatment for earlier stage individuals with anxiety.

\section{Pharmacologic Recommendations}

An SSRI drug is the preferred pharmacologic option for treatment of anxiety when it occurs either as an isolated symptom or when coexisting depression or obsessive perseverative behaviors are present.

Warning should be given of potential short-term exacerbation of anxiety when an SSRI is initiated. If exacerbation occurs it may be appropriate to add a short-term course (one or two weeks) of a benzodiazepine.

Alternative serotonergic drugs (SSRI, NSRI, clomipramine) are pharmacologic options if the initial SSRI is ineffective or not tolerated.

Mirtazapine is a pharmacologic option particularly if coexisting sleep disorder is present.

An antipsychotic is a pharmacologic option particularly if needed for treatment of coexisting chorea.

Clomipramine is a pharmacologic option particularly if needed for coexisting obsessive perseverative behaviors.

Long-term use of a benzodiazepine drug is discouraged in ambulatory individuals with HD unless all other options have failed.

\section{Anxiety in HD: Consensus definition and clinical} description

Anxiety in HD may include symptoms of general anxiety, social anxiety, anticipatory anxiety, panic, and post-traumatic stress disorder [19]. Anxiety often co-exists with depression [1, 4] and may be triggered by external environmental factors including unfamiliar situations, changes in routine, physical discomfort, or performance anxiety due to cognitive and physical impairments. Anxiety can occur in each clinical stage of HD, including the prodromal stage (before the motor diagnosis). With disease progression, it may manifest as restlessness due to difficulty in verbally communicating distress. Anxiety can worsen chorea in HD.

Experts agreed that anxiety in HD can sometimes be confused with akathisia, which is a possible side effect of some drugs (e.g., antipsychotics or tetrabenazine) commonly used to treat symptoms of HD. There was consensus that anxiety is a treatable symptom in HD. Modification of external environmental factors contributing to anxiety in HD may be a key step in management and cognitive behavioral therapy may be helpful particularly in prodromal and early stages. Anxiety is often amenable to treatment using available drugs. Many of these drugs can also be effective against co-existing symptoms such as depression or perseverative behaviors (Table 3 ). 
Table 4

\section{Clinical Practice Guidelines for Apathy in HD}

\section{General Recommendations}

Differentiate apathy from impaired ability of the individual to perform motor or cognitive tasks.

Consider dose reduction of medications (prescribed for other symptoms) which may contribute to apathy.

Treat coexisting depression that may contribute to apathy.

\section{Behavioral Recommendations}

Provide prompts and encouragement of social and physical activities that have been adapted to the individual.

\section{Pharmacologic Recommendations}

An antidepressant is the preferred pharmacologic option when there is difficulty differentiating apathy of depression from apathy of HD.

Consider a trial of an activating antidepressant or stimulant drug as pharmacologic options for the non-depressed individual.

Warning should be given for potential worsening of irritability and sleep disturbance when prescribing an activating antidepressant or a stimulant drug.

\section{Apathy in HD: Consensus definition and clinical} description

Apathy in HD is characterized by lack of motivation and diminished goal directed activities in three domains: behavior (lack of initiative or depending on prompts), cognitive (lack of interests or lack of concern), and emotion (constricted affect or lack of emotional responsiveness) [4]. In general, it can be distinguished from depression by the lack of sadness and negative or suicidal thoughts [20]. Apathy must be differentiated from delayed responses due to slowed cognition, speech latency, reduced or impaired motor initiation, and/or an inability to participate due to physical impairment. Apathy may occur before the onset of motor symptoms, and it often increases with advancing clinical stage of disease $[9,21,22]$.

Experts agreed that an environment that includes regularly scheduled social and physical activities adapted to the individual may be a beneficial treatment of apathy syndrome in HD (Table 4). A dose reduction of medications (e.g., SSRIs, neuroleptics) prescribed for other symptoms of HD should be considered (Table 4) before new drugs with questionable efficacy and potential undesirable side effects are prescribed.

\section{Psychosis in HD: Consensus definition and clinical description}

Psychosis in HD is defined by the presence of prominent delusions and/or hallucinations. Although the prevalence of psychosis in HD is usually considered relatively low compared to other neuropsychiatric symptoms, several of the surveyed experts used the free text to note that prevalence of psychosis in HD is likely higher than reported. Respondents emphasized that a low level of psychotic symptoms may be easily missed, occur transiently, masked if an antipsychotic is used for another indication, or remain undiagnosed at advanced stages of disease when ability to communicate is impaired.

While co-morbid schizophrenia spectrum or mood disorders with psychotic features may be considered in the differential diagnosis, psychotic symptoms may also occur in HD due to infections, metabolic 
Table 5

\section{Clinical Practice Guidelines for Psychosis in HD}

\section{General Treatment Recommendations}

Identify and treat comorbid medical conditions that can precipitate acute onset of psychotic symptoms that include infectious, metabolic, toxic, drug-related, substance use, or other medical or acute psychosis/delirium.

Treat co-existing psychiatric symptoms of HD including obsessive perseverative and sleep disorders.

Modify external environmental factors that may contribute to distress of psychotic symptoms.

\section{Pharmacologic Recommendations}

An antipsychotic drug is the first line pharmacologic treatment for psychosis in HD.

An alternative antipsychotic should be used when psychotic symptoms have not been adequately controlled by the initial drug.

Exceeding maximum recommended dose of any antipsychotic is discouraged.

Combining antipsychotic drugs is discouraged, reserved only for more severe presentations of psychosis in HD.

Consider clozapine when psychotic symptoms have not adequately responded to other antipsychotics in those situations where interval blood testing is possible.

Regularly reassess the continued need of an antipsychotic because many of the adverse effects of these drugs are difficult to distinguish from aspects of disease progression.

issues, substance use, or other medical causes of psychosis/delirium. It may also be clinically difficult to distinguish delusions from perseverative symptoms.

The experts agreed that the side effect profiles of antipsychotics should be considered when choosing an antipsychotic drug in HD (Table 5). However, while a majority (83\%) indicated they prefer a second-generation antipsychotic, a significant number preferred a first-generation alternative when chorea dominates. Clozapine may be considered as a treatment for psychosis in HD when symptoms have not responded to adequate trials of other antipsychotic drugs in those situations where interval blood tests to monitor for agranulocytosis is feasible.
Sleep disorders in HD: Consensus definition and clinical description

Sleep disturbances may increase in severity with advancing clinical stage of disease. Sleep disorders in HD include increased sleep latency (difficulty falling asleep), decreased sleep efficiency (difficulty maintaining sleep), early awakening, disrupted day-night cycles and excessive daytime sleepiness. Circadian rhythm disruption is also a prominent feature of sleep disorders in HD. It was agreed that coexisting symptoms including anxiety and depression can contribute to sleep impairment; the presence of chorea can interfere with onset of sleep in HD. Conversely, poor sleep quality can negatively impact other disease symptoms including depression, anxiety, irritability, 
Table 6

\section{Clinical Practice Guidelines for Sleep Disorders in HD}

\section{General recommendations}

Treat co-morbid medical conditions, coexisting psychiatric symptoms, pain, or substance use that can contribute to sleep disturbance in HD.

Assess and adjust dosing schedule of drugs that may contribute either to daytime sleepiness or nocturnal insomnia.

\section{Behavioral Treatment Recommendations}

Provide information regarding good sleep hygiene as the initial step for treating sleep disturbances.

\section{Pharmacologic Treatment Recommendations}

Melatonin is a pharmacologic option particularly when there is pattern of circadian rhythm disordered sleep.

Sedating antidepressants such as mirtazapine or trazodone are pharmacologic options for treating sleep disorders in HD.

Sedating neuroleptics such as olanzapine and quetiapine are pharmacologic options for treating sleep disorders in HD.

Clomipramine is a pharmacologic option if this drug is needed for management of coexisting obsessive perseverative symptoms.

Use of a benzodiazepine is discouraged in ambulatory individuals unless all other options have failed.

and apathy, and can further impair cognitive and functional capacities in HD.

The experts agreed that the choice of drug for treating sleep impairment in HD is often dependent on presence of coexisting disease symptoms and may vary with stage of disease (Table 6). However, they noted that drugs used in the treatment of sleep disorders in HD may be associated with adverse effects that mimic symptoms of HD including day time sleepiness, fatigue, apathy, slowing of cognitive processing, and worsening of balance leading to falls.

\section{DISCUSSION}

We present clinical practice recommendations and management guides based on a rigorous guideline development process. Recommendations include guidance for both the evaluation and management of agitation, anxiety, apathy, psychosis and sleep disorders associated with HD. While many of the recommendations are like those contained in general psychiatric guidelines and guidelines for other disease areas (e.g., Alzheimer's disease [23] Parkinson's disease [24]), the present work is intended to inform clinicians, scientists, patients and carers on the management of these neuropsychiatric symptoms specifically in HD. As with all guidelines, clinical judgment is of paramount importance for each unique patient.

There were commonalities across all five symptom domains considered, and these can be considered 'general principles' in the management of neuropsychiatric symptoms in HD. For example, it is recommended to obtain information from both the patient and the carer, as it is well known that people with HD may have impaired awareness of their condition $[25,26]$. As in any other neurologic and/or psychiatric illness, the identification and treatment of co-morbid medical conditions and possible environmental triggers are always key factors to address in the management of all neuropsychiatric symptoms associated with HD. The recognition and prompt treatment of neuropsychiatric symptoms as a preventative strategy for development of more severe 
symptoms is also important. The management of neuropsychiatric symptoms in HD can be complex because symptoms often co-exist and treatment decisions should be adapted to cover all symptoms while limiting polypharmacy. Non-pharmacologic interventions, e.g., addressing environmental factors or behavioral interventions, should be considered first. When pharmacologic agents are required, one should be aware of possible pharmacodynamic interactions. It is advised to first use medications with the least unwanted adverse effects, as medication side-effects may be difficult to differentiate from or worsen cognitive and motor symptoms of the disease. It is therefore important to regularly reevaluate the treatment regimen of any drug given for neuropsychiatric symptoms. This is also important because treatment may vary in different stages of disease. We also recommend consultation with a psychiatrist who has knowledge of HD when neuropsychiatric symptoms are resistant to standard treatment. Another important general recommendation is to provide patients and carers with accessible information regarding their specific neuropsychiatric symptoms. Such information can help build the therapeutic alliance, improve understanding and symptom reporting, and optimize a treatment plan [27].

While strong agreement was readily reached for most statements evaluated in the first round, there were some important areas of contention that merit further investigation. For example, there was a lack of consensus regarding the observed prevalence of agitation, anxiety, apathy and psychosis in HD relative to disease stage. This is in line with the widely varying prevalence estimates of neuropsychiatric symptoms reported in the literature. While some studies describe psychotic symptoms in early stages of disease [28], others report a more common occurrence in individuals with advancing stage of disease $[29,30]$. Differences in opinion may therefore also reflect a bias of the types of setting the respondents practice in, with respondents working in nursing homes potentially seeing a higher prevalence of psychotic symptoms than clinicians working with early stage patients. Likewise, although more consistently associated with disease progression $[9,22,31]$, the reported prevalence of apathy has ranged from $32 \%$ to $76 \%[1,2,9,32,33]$. This may be due to variation in assessments used. For these statements, consensus was eventually reached by removal of the word 'common' when referring to the prevalence of these symptoms. Other sources of contention were in relation to the management of beta-blockers for anxiety, use of physical restraints in patients exhibiting aggressive symptoms, use of benzodiazepines for sleep disorders and the management of anxiety.

It is important to be aware that our guidelines are based on the opinions of 84 clinicians experienced in the management of neuropsychiatric symptoms of HD. At present, the only evidence-based guidelines for HD are for the management of chorea [34]. The American Academy of Neurology produced one such guideline which was strongly criticized as being of low grade evidence and for providing clinical recommendations that do not represent current clinical practice. This guideline was based on HD trials (and trial endpoints) that have often been designed to answer questions leading to drug approval rather than clinical practice [35]. This is a particular problem in HD which, due to its low prevalence, lacks the necessary randomized clinical trial evidence required to document the efficacy and safety of pharmacologic and non-pharmacologic interventions. Indeed, the current literature is skewed to modern agents under development for HD, and there may never be enough information for the older drugs to meet the rigors of evidence-based medicine.

Expert-led guidelines can help overcome these barriers and we utilized a modified IOM [14] approach to systematically consider the different aspects of symptom management. While we believe the survey questions were comprehensive, it was not feasible to try to cover every possibility in this complex disease. Indeed, although the experts completed surveys about isolated symptoms, a key strength of the pharmacologic recommendations is that they purposefully take co-existing symptoms into account. The experts were also given the opportunity to give comments in free text and such comments were considered by the expert committee when combining and summarizing the clinical recommendations. For example, while the use of benzodiazepines was not initially addressed in the sleep survey, the committee decided to include a cautionary statement ("Use of a benzodiazepine is discouraged in ambulatory individuals unless all other options have failed") for sleep disorders because the committee agreed that benzodiazepines are overused in treating sleep disorders in HD. Another limitation of the survey is that not all statements had the same number of respondents, for example 21 of the 83 experts (25\%) involved in the anxiety survey indicated that they had insufficient experience to comment on the use of clomipramine. However, strong agreement was reached among the other 62 experts that clomipramine can be useful 
in the setting of perseverative behaviors when treatment with a SSRI has failed. While the selection of experts was not systematic, they were drawn from investigators involved in the large Enroll-HD platform which has been specifically designed to support an international and expert approach to HD research [18]. Finally, as with all surveys, we cannot rule out the potential bias introduced by the self-selection of respondents who agreed to participate or any recall bias that may have impacted the accuracy of the experts' answers.

In summary, randomized controlled trials are needed to study the best treatment options for the various neuropsychiatric symptoms associated with HD. In the absence of such high-level evidence, the proposed expert-based algorithms presented herein have been developed to guide the clinical management of agitation, anxiety, apathy, psychosis, and sleep disorders in HD. Although not specifically tested in the HD population, clinical experience indicates that most of the neuropsychiatric symptoms discussed are treatable using pharmacologic and non-pharmacologic strategies developed for use in other populations. Treatment of the symptoms as experienced in HD generally follows the same recommendations as in other populations. However, the management of neuropsychiatric symptoms in HD is often more complex because symptoms often co-exist and treatment decisions should be adapted to cover all symptoms while limiting polypharmacy.

\section{ACKNOWLEDGMENTS}

Funding for this work, including author stipends, was obtained from the Griffin Foundation and Auspex Pharmaceuticals. Neither funding organization was involved in drug development for any of the symptoms included in this project. We thank Anita Chadha-Patel (ACP Clinical Communications Ltd) who received non-restricted Griffin Foundation and Auspex Pharmaceuticals funding for medical writing assistance.

\section{CONFLICT OF INTEREST}

KEA reports consultancy for Teva, Roche, CHDI Foundation, Prana and Lundbeck; participation in studies funded by Teva and Vaccinex, and research funding from Cures Within Reach. EVD and NG have no further disclosures to report. CTL was supported by an Australian National Health and Medical Research Council- Australian
Research Council Dementia Research Development Fellowship (APP1107657). LVG reports consultancy for Teva and Lundbeck and speaker fees from Teva.

\section{SUPPLEMENTARY MATERIAL}

The supplementary material is available in the electronic version of this article: http://dx.doi.org/ 10.3233/JHD-180293.

\section{REFERENCES}

[1] Craufurd D, Thompson JC, Snowden JS. Behavioral changes in Huntington disease. Neuropsychiatry Neuropsychol Behav Neurol. 2001;14:219-26.

[2] Paulsen JS, Ready RE, Hamilton JM, Mega MS, Cummings JL. Neuropsychiatric aspects of Huntington's disease. J Neurol Neurosurg Psychiatry. 2001;71:310-4.

[3] van Wamelen DJ, Roos RA, Aziz NA. Therapeutic strategies for circadian rhythm and sleep disturbances in Huntington disease. Neurodegener Dis Manag. 2015;5:549-59.

[4] Kingma EM, van Duijn E, Timman R, van der Mast RC, Roos RA. Behavioural problems in Huntington's disease using the Problem Behaviours Assessment. Gen Hosp Psychiatry. 2008;30:155-61.

[5] Rickards H, De Souza J, van Walsem M, van Duijn E, Simpson SA, Squitieri F, et al. Factor analysis of behavioural symptoms in Huntington's disease. J Neurol Neurosurg Psychiatry. 2011;82:411-2.

[6] Naarding P, Kremer HP, Zitman FG. Huntington's disease: A review of the literature on prevalence and treatment of neuropsychiatric phenomena. Eur Psychiatry. 2001;16: 439-45.

[7] Gray MA, Egan GF, Ando A, Churchyard A, Chua P, Stout JC, et al. Prefrontal activity in Huntington's disease reflects cognitive and neuropsychiatric disturbances: The IMAGEHD study. Exp Neurol. 2013;239:218-28.

[8] Ondo WG, Tintner R, Thomas M, Jankovic J. Tetrabenazine treatment for Huntington's disease-associated chorea. Clin Neuropharmacol. 2002;25:300-2.

[9] van Duijn E, Reedeker N, Giltay EJ, Roos RA, van der Mast RC. Correlates of apathy in Huntington's disease. J Neuropsychiatry Clin Neurosci. 2010;22:287-94.

[10] Bonelli RM, Wenning GK. Pharmacological management of Huntington's disease: An evidence-based review. Curr Pharm Des. 2006;12:2701-20.

[11] Mestre T, Ferreira J, Coelho MM, Rosa M, Sampaio C. Therapeutic interventions for symptomatic treatment in Huntington's disease. Cochrane Database Syst Rev. 2009; CD006456.

[12] Mestre TA, Ferreira JJ. An evidence-based approach in the treatment of Huntington's disease. Parkinsonism Relat Disord. 2012;18:316-20.

[13] Gelderblom H, Wustenberg T, McLean T, Mutze L, Fischer W, Saft C, et al. Bupropion for the treatment of apathy in Huntington's disease: A multicenter, randomised, double-blind, placebo-controlled, prospective crossover trial. PLoS One. 2017;12:e173872.

[14] Graham R, Mancher M, Miller Wolman D, Greenfield S, Steinberg E. Clinical Practice Guidelines We Can Trust. Washington, DC; 2011. 
[15] Burgunder JM, Guttman M, Perlman S, Goodman N, van Kammen DP, Goodman L. An international survey-based algorithm for the pharmacologic treatment of chorea in Huntington's disease. PLoS Curr. 2011;3:RRN1260.

[16] Groves M, van Duijn E, Anderson K, Craufurd D, Edmondson MC, Goodman N, et al. An international survey-based algorithm for the pharmacologic treatment of irritability in Huntington's disease. PLoS Curr. 2011;3:RRN1259.

[17] Anderson K, Craufurd D, Edmondson MC, Goodman N, Groves M, van Duijn E, et al. An international survey-based algorithm for the pharmacologic treatment of obsessivecompulsive behaviors in Huntington's disease. PLoS Curr. 2011;3:RRN1261.

[18] Landwehrmeyer GB, Fitzer-Attas CJ, Giuliano JD, Gonçalves N, Anderson KE, Cardoso F, et al. Data Analytics from Enroll-HD, a Global Clinical Research Platform for Huntington's Disease. Mov Disord Clin Pract. 2016;4: 212-24.

[19] Pflanz S, Besson JA, Ebmeier KP, Simpson S. The clinical manifestation of mental disorder in Huntington's disease: A retrospective case record study of disease progression. Acta Psychiatr Scand. 1991;83:53-60.

[20] Levy ML, Cummings JL, Fairbanks LA, Masterman D, Miller BL, Craig AH, et al. Apathy is not depression. J Neuropsychiatry Clin Neurosci. 1998;10:314-19.

[21] Thompson JC, Snowden JS, Craufurd D, Neary D. Behavior in Huntington's disease: Dissociating cognition-based and mood-based changes. J Neuropsychiatry Clin Neurosci 2002; 14:37-43.

[22] Burns A, Folstein S, Brandt J, Folstein M. Clinical assessment of irritability, aggression, and apathy in Huntington and Alzheimer disease. J Nerv Ment Dis. 1990;178:20-6.

[23] APA Work Group on Alzheimer's Disease and other Dementias, Rabins PV, Blacker D, Rovner BW, Rummans T, Schneider LS, et al. American Psychiatric Association practice guideline for the treatment of patients with Alzheimer's disease and other dementias. Second edition. Am J Psychiatry. 2007;164:5-56.

[24] Miyasaki JM, Shannon K, Voon V, Ravina B, KleinerFisman G, Anderson K, et al. Practice Parameter: Evaluation and treatment of depression, psychosis, and dementia in Parkinson disease (an evidence-based review): Report of the
Quality Standards Subcommittee of the American Academy of Neurology. Neurology. 2006;66:996-1002.

[25] McCusker E, Loy CT. The many facets of unawareness in huntington disease. Tremor Other Hyperkinet Mov (NY). 2014;4:257.

[26] Sitek EJ, Thompson JC, Craufurd D, Snowden JS. Unawareness of deficits in Huntington's disease. J Huntingtons Dis. 2014;3:125-35.

[27] Summers RF, Barber JP. Therapeutic alliance as a measurable psychotherapy skill. Acad Psychiatry. 2003;27: 160-5.

[28] Rosenblatt A, Leroi I. Neuropsychiatry of Huntington's disease and other basal ganglia disorders. Psychosomatics. 2000;41:24-30.

[29] Marder K, Zhao H, Myers RH, Cudkowicz M, Kayson E, Kieburtz K, et al. Rate of functional decline in Huntington's disease. Huntington Study Group. Neurology. 2000;54: 452-8.

[30] van Duijn E, Craufurd D, Hubers AA, Giltay EJ, Bonelli R, Rickards H, et al. Neuropsychiatric symptoms in a European Huntington's disease cohort (REGISTRY). J Neurol Neurosurg Psychiatry. 2014;85:1411-8.

[31] Caine ED, Shoulson I. Psychiatric syndromes in Huntington's disease. Am J Psychiatry. 1983;140:728-33.

[32] Kulisevsky J, Litvan I, Berthier ML, Pascual-Sedano B, Paulsen JS, Cummings JL. Neuropsychiatric assessment of Gilles de la Tourette patients: Comparative study with other hyperkinetic and hypokinetic movement disorders. Mov Disord. 2001;16:1098-104.

[33] Baudic S, Maison P, Dolbeau G, Boisse MF, Bartolomeo P, Dalla Barba G, et al. Cognitive impairment related to apathy in early Huntington's disease. Dement Geriatr Cogn Disord. 2006;21:316-21.

[34] Armstrong MJ, Miyasaki JM, American Academy of N. Evidence-based guideline: Pharmacologic treatment of chorea in Huntington disease: Report of the guideline development subcommittee of the American Academy of Neurology. Neurology. 2012;79:597-603.

[35] Reilmann R. Pharmacological treatment of chorea in Huntington's disease-good clinical practice versus evidencebased guideline. Mov Disord. 2013;28:1030-33. 